\title{
Resveratrol Attenuated Low Ambient Temperature-Induced Myocardial Hypertrophy via Inhibiting Cardiomyocyte Apoptosis
}

\author{
Kun Yin ${ }^{a}$ Liang Zhao Dan Feng ${ }^{a}$ Wenya Ma ${ }^{a}$ Yu Liu ${ }^{a}$ Yang Wanga Jing Liang ${ }^{a}$ \\ Fan Yang ${ }^{a}$ Chongwei Bia Hongyang Chen ${ }^{a}$ Xingda Li ${ }^{a}$ Yanjie Lu ${ }^{a, b}$ Benzhi Cai ${ }^{a, c}$ \\ aDepartment of Pharmacology (Key Laboratory of Cardiovascular Research, Ministry of Education), \\ Harbin Medical University, Harbin, 'Institute of Cardiovascular Research, Harbin Medical University, \\ Harbin, 'Northern Translational Medicine Cooperative Research Center, Heilongjiang Academy of \\ Medical Sciences, Harbin, China
}

\section{Key Words}

Low ambient temperature - Myocardial hypertrophy B Blood pressure $\cdot$ Cardiac function • Mitochondria

\begin{abstract}
Background/Aims: Low ambient temperature is an important risk factor for cardiovascular diseases, and has been shown to lead to cardiac hypertrophy. In this study, we aim to investigate if Resveratrol may inhibit cold exposure-induced cardiac hypertrophy in mice, and if so to clarify its molecular mechanism. Methods: Adult male mice were randomly assigned to Control group (kept at room temperature), Cold group (kept at low air temperature range from $3^{\circ} \mathrm{C}$ to $5^{\circ} \mathrm{C}$ ) and Resveratrol treatment group (100mg/kg/day) for eight weeks. HE staining, Masson staining and Transmission electron microscopy were employed to detect cardiac structure, fibrosis and myocardial ultrastructure, respectively. Echocardiogram was used to measure myocardial functions. Western blot was used to detect the expression of MAPK pathway and apoptotic proteins. TUENL assay was performed to evaluate cardiomyocyte apoptosis. qRT-PCR was employed to measure the mRNA level. Results: Cold-treated mice showed a higher heart/body weight ratio and heart weight/tibia length ratio compared with control mice, and Resveratrol treatment may suppress these changes in cold-treated mice. Myocardial cross-section area and cardiac collagen volume were larger in cold group than control group, which also can be attenuated by Resveratrol treatment. Also, Resveratrol improved the ultrastructure damage of myocardium such as myofibril disarray in cold group. Echocardiogram measurement showed that EF and FS values in cold group declined apparently as compared to control group, and Resveratrol may improve the reduction of heart functions. The expression of p-JNK, p-p38 and p-ERK relative to total JNK, p38 and ERK in cold group was not altered in cold group and
\end{abstract}

$\mathrm{K}$ Yin, L. Zhao and D. Feng made equal contributions to this work.

Prof. Benzhi Cai

KARGER 125 
Resveratrol group as compared to control group. Cold-treated mouse hearts also showed the upregulation of hypertrophy-related miRNA-miR-328 but not miR-23a, and Resveratrol treatment can inhibit the increase of miR-328. Finally, Resveratrol treatment also may suppress apoptosis of myocardium in cold-treated mouse hearts via inhibiting Bax and caspase- 3 activation. Conclusion: In summary, low ambient temperature can cause enlarged heart, ultrastructure damage of myocardium and weakened functions, and Resveratrol treatment effectively suppressed these changes at least partially via inhibiting cardiomyocyte apoptosis.

Copyright @ 2015 S. Karger AG, Basel

\section{Introduction}

Cardiovascular disease is one of the three major causes of death all over the world, and its morbidity and mortality display an upward trend year by year. In recent years, more and more researchers pay attention to the impact of low ambient temperature on the risk of cardiovascular diseases [1-3]. Increasing evidence showed that there is a striking relationship between cold temperature and cardiovascular mortality [1, 3-6]. Exposure to cold temperature has been reported to contribute to the increased incidence of cardiovascular deaths, and especially younger and hyperglycemic patients are more susceptible to cold temperature [1]. Epidemical investigation also showed that people living in cold region has a higher incidence of heart diseases and hypertension [1,3]. So, low ambient temperature stress is considered as a new potential risk factor of sudden cardiovascular death in human beings [5, 7-9]. Animal studies also clarified that low ambient temperature exposure is able to induce and aggravate myocardial hypertrophy and hypertension, which are attributed to neuroendocrine, oxidative stress, endothelial function, inflammation, AMP-activated protein kinase (AMPK), c-myc, capsaicin receptor transient receptor potential vanilloid 1 (TRPV1), etc [8, 10-13]. For example, heart-specific inhibition of c-Myc expression was shown to attenuate cold-induced cardiac hypertrophy [12]. Angiotensin II type-1 A (AT1A) receptor gene knockout also can abolish low temperature-induced increase of blood pressure partially by suppressing NO system [14]. The inhibition of mineralocorticoid receptor (MR) using short hairpin small interference siRNA significantly attenuated cold-induced hypertension and renal damage [15]. So, the inhibition of low temperature-induced cardiac remodeling is considered as an important approach for decreasing the high mortality of cardiovascular diseases in patients living in cold regions and in winter.

Resveratrol is one kind of the antioxidant compound and widely exists in peanuts, berries, grapes and some China herbs [16-18]. Recently, more and more studies uncover that Resveratrol has anticancer actions on a variety of cancers [19]. Resveratrol was shown to induce cell death in cervical cancer cells through apoptosis and autophagy [19]. An antitumor activity of Resveratrol was identified in human colorectal cancer cell lines through calorie-restriction pathway [20]. Resveratrol treatment also may suppress tumor cell growth in lung cancer cells via inducing premature senescence and ROS-mediated DNA damage [21]. Besides, Resveratrol can protect hearts against cardiovascular diseases. For example, Resveratrol was shown to suppress left ventricular hypertrophy and heart failure $[17,22]$. Resveratrol treatment also inhibits ischemia/reperfusion injury and ventricular arrhythmias [23]. Apoptosis of cardiomyocytes was also protected by Resveratrol treatment. However, whether Resveratrol treatment can protect myocardium against cold exposureinduced hypertrophy remained unknown. The present study was designed to clarify this notion.

\section{Material and Methods}

Animals

The healthy Kunming adult male mice were purchased from the Animal Center of Affiliated Second Hospital of Harbin Medical University, and then housed under the condition of temperature $23 \pm 1^{\circ} \mathrm{C}$ and 


\section{Cellular Physiology Cell Physiol Biochem 2015;35:2451-2462 \begin{tabular}{l|l|l}
\hline DOI: 10.1159/000374045 & C 2015 S. Karger AG, Basel
\end{tabular} \begin{tabular}{l|l} 
and Biochemistry Published online: April 24, 2015 & www.karger.com/cpb
\end{tabular} \\ Yin et al.: Resveratrol Inhibited Cold-Induced Heart Hypertrophy}

humidity 55-60\%. Mice were maintained on a 12 hours dark-light artificial cycle, with food and water ad libitum. The animal protocol was approved by the Institutional Animal Care and Use Committee of Harbin Medical University. All the procedures were in agreement with the Directive 2010/63/EU of the European Parliament. According to the methods described previously [14], mice were randomly divided into three groups: Control group, Cold group, and Resveratrol (Sigma Chemical Co., USA) treatment group. Control group mice were kept in room temperature, and Cold group mice was continually exposed to low ambient temperature $3-5^{\circ} \mathrm{C}$ in a cold chamber for eight weeks. For Resveratrol treatment group, cold-treated mice were orally administrated everyday by Resveratrol $100 \mathrm{mg} / \mathrm{kd} /$ day at the same time. Oral administration of Resveratrol was performed by a 20-G ballpoint oral feeding needle (Popper and Sons, New Hide Park, NY). Eight weeks after cold exposure, mice in three groups were sacrificed and ventricular myocardium was collected for further experiments.

Echocardiography

After the mice were anesthetized with ketamine $(100 \mathrm{mg} / \mathrm{kg})$ and xylazine $(10 \mathrm{mg} / \mathrm{kg})$, echocardiography examination was performed according to the methods described previously [24, 25]. Cardiac functions were measured using a HP Sonos 2500 echocardiographic system (Hewlett-Packard, New Orleans, LA, USA) equipped with a 30-MHz phased-array transducer. Ejection fraction (EF), Fraction shortening (FS), Diastolic left ventricular (LV) posterior wall thickness (LVPWd), Systolic left ventricular posterior wall thickness (LVPWs), Left ventricular end-diastolic dimensions (LVIDd) and Left ventricular end-systolic dimensions (LVIDs) were acquired using M-mode acquisitions. Ejection fraction (EF) was calculated from the M-mode LV dimensions as described previously [26]. Parameters for the calculation of heart function were measured from 3 consecutive systole-diastole cycles, and were analyzed by an echocardiography expert and a cardiologist. Left ventricular EF was calculated as EF = (EDV-ESV)/EDV.

\section{Blood Pressure}

The blood pressure of mice from each group was invasively measured using the tail-cuff method according to previous study [27]. Systolic blood pressure (SBP) was measured in mice by BP-2000 Blood Pressure Analysis System (Visitech Systems, Cary, NC). Mice from each group were moved into an individual rodent restraint holder, and then SBP were recorded 10 times in rapid succession. Afterward, the obtained results will be averaged and analyzed. The standard deviation $<10 \mathrm{mmHg}$ of SBP will be accepted for further analysis.

\section{HE and Masson staining}

When mice in each group were treated with the indicated condition for eight weeks, mice in each groups were sacrificed and the ventricle tissues were collected. The collected hearts were fixed in $4 \%$ formalin in PBS, and then embedded in paraffin. Left ventricle tissue from the embedded hearts was sectioned into 5- $\mu \mathrm{m}$, and then underwent Hematoxylin-Eosin (HE) staining and Masson staining according to the method described previously [24]. Samples on the section were analyzed under a light microscopy. The cross-sectional area of cardiomyocytes was measured and calculated using the Image-pro-plus 6.0. The fraction of myocardial volume occupied by fibrillar collagen was calculated as the percent total surface area occupied by the interstitial fibrosis.

\section{TUENL staining}

Terminal deoxynucleotidyl transferase dUTP nick end labeling (TUNEL) staining was used to detect the apoptosis of myocardium from each group by TUNEL detection kit (Roche, Penzberg, Germany) according to the manufacturer's protocol. The sectioned heart tissues were fixed with $4 \%$ paraformaldehyde in PBS for $1 \mathrm{~h}$ at room temperature, and permeabilized with $0.1 \%$ Triton X-100 in $0.1 \%$ sodium citrate for 2 min on ice. After washing in PBS, sections were incubated with the TUNEL reaction mixture for $1 \mathrm{~h}$ at $37{ }^{\circ} \mathrm{C}$. After washing with PBS, the stained cells were visualized using a fluorescence microscopy (Eclipse TE300, Nikon, Japan).

\section{Transmission electron microscopy}

Ultrastructure of myocardium from each group was visualized by a Hitachi H-7650 Transmission electron microscopy (TEM). The effects of Resveratrol on myofibril and mitochondrial morphological alterations of cold-treated mouse hearts were analyzed. 


\section{Cellular Physiology Cell Physiol Biochem 2015;35:2451-2462 \begin{tabular}{l|l} 
and Biochemistry Published online: April 24, 2015 & $\begin{array}{l}\text { C 2015 S. Karger AG, Basel } \\
\text { www.karger.com/cpb }\end{array}$ \\
\hline
\end{tabular} \\ Yin et al.: Resveratrol Inhibited Cold-Induced Heart Hypertrophy}

qRT-PCR

Total RNA was extracted from heart tissues using Trizol reagent (Invitrogen, USA) according to the manufacturer's instruction. The level of miR-328 and miR-23a was measured using SYBR Green PCR Master Mix Kit (Applied Biosystems, Foster City, CA, USA) with U6 as an internal control. The miR-328 and miR23a levels were determined on a 7500 FAST Real-Time PCR System (Applied Biosystems). The method to quantify the miR-328 level was as previously described [28]. For measuring miR-23a, the primers forward, 5'-GGATCACATTGCCAGGGAT-3', reverse, 5'-CAGTGCGTGTCGTGGAGT-3' were used; U6 was used as an internal control. The relative expression of miRNAs was quantified using comparative $2^{-\Delta \Delta C t}$ method.

\section{Western blot analysis}

Myocardial protein samples were extracted from left ventricle of mice in each group. For the detection of the expression of p-JNK, p-p38, p-ERK, T-JNK, T-p38 and T-ERK, the left ventricle samples were collected from the mice exposed to cold for 6 hours. The protein samples (20-50 $\mu \mathrm{g})$ were fractionated by SDSPAGE (10\%-12\% polyacrylamide gels) and transferred to PVDF membrane (Millipore, Bedford, MA). The blots were probed with primary antibody including p-JNK, p-p38, p-ERK, T-JNK, T-p38, T-ERK (Abcam, Cambridge, MA), Bcl-2, Bax, cleaved caspase-3, and GAPDH (Santa Cruz, USA) and then with secondary antibody (Alexa Fluor). The blots were scanned by Odyssey Infrared Imaging System (LI-COR Bioscience, Lincoln, NE). Western blot bands were quantified using Odyssey v1.2 software. The results were expressed as fold changes normalized to GAPDH as an internal control.

\section{Data analysis}

Data are presented as mean \pm SEM. Statistical analysis was performed by using GraphPad Prism 5.0 (GraphPad Software Inc., San Diego, CA). Two-tailed Student'.s t-test or Newman-Keuls Multiple Comparison Test were applied for comparisons between groups. $\mathrm{P}<0.05$ and were considered as statistically difference.

\section{Results}

\section{Resveratrol inhibited cold-induced alteration of cardiac structure}

Firstly, we observed the effects of Resveratrol on myocardial structure in mice subjected to cold exposure. As shown in Figure 1A-1C, cold-treated mice showed a significant increase of heart weight/body weight ratio and heart weight/tibia length ratio compared with control mice $(n=6, p<0.05)$. But Resveratrol $(100 \mathrm{mg} / \mathrm{kg} /$ day $)$ treatment can obviously inhibit the increase of heart/body index and heart weight/tibia length ratio in cold-treated mice $(n=6$, $\mathrm{p}<0.05)$. It suggests that Resveratrol may inhibit cold-induced myocardial hypertrophy in mice. Moreover, HE staining displayed a significant increase of cross-section area of ventricular myocardium from cold-treated mice compared with control mice, which also can be partially reversed by Resveratrol $(100 \mathrm{mg} / \mathrm{kg} /$ day) treatment (Fig. 2A). Masson staining showed that cardiac fibrosis was markedly increased in cold-treated mice as well, but Resveratrol treatment may inhibit cardiac fibrosis in cold-treated mice (Fig. 2B). All these findings suggest that Resveratrol can improve cardiac structure in mice subjected to cold exposure.

\section{Resveratrol improved ultrastructure of myocardium in cold-treated mice}

Myocardial remodeling observed in cold-treated mice implies intrinsic cellular damages such as mitochondrial dysfunction in ventricular myocytes. To test if cellular ultrastructure was damaged in myocardium of cold-treated mice, transmission electron microscopy was employed to visualize myofibril and mitochondrial morphological alterations. As shown in Figure 3, the myocardium from cold-treated mice displayed the loss of sarcomere, myofibrillar disarray and mitochondrial vacuolation as compared to the characteristic linear array of sarcomeres and myofibrils in control mice. Distinct morphological features including disarrayed sarcomere and swollen mitochondria were reduced in cold-treated mice with Resveratrol treatment (Fig. 3). 
Fig. 1. Resveratrol inhibited cold exposure-induced heart enlargement in mice. (A), The representative pictures of hearts from control group, cold group and Resveratrol group. (B, C), An increase of heart weight/body weight ratio and heart weight/tibia length ratio in cold-treated mice was obviously inhibited by Resveratrol treatment. The experiments were repeated three times. $n=6$ mice in each group. Ctl, Control mice; Model, Cold-treated mice; Rsv, Cold-treated mice with Resveratrol supplement. ${ }^{*} \mathrm{p}<0.05$ vs Control group, \# $\mathrm{p}<0.05$ vs Cold group.
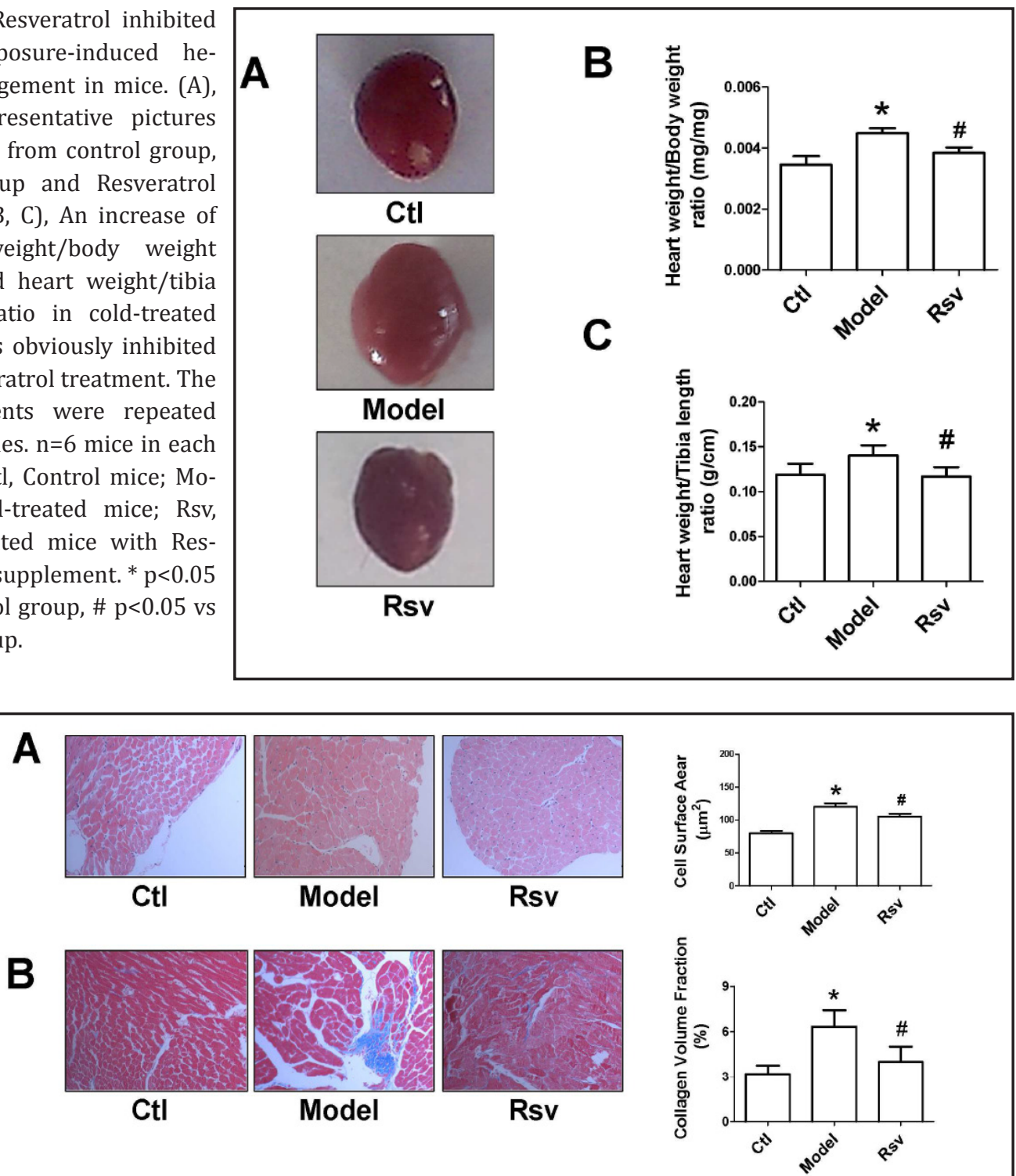

Fig. 2. Resveratrol attenuated cold exposure-induced myocardial structural remodeling. (A), HE staining displayed that cold exposure caused a significant increase of cross-section area of ventricular myocardium, which also can be partially prevented by Resveratrol treatment. (B), Cold exposure markedly increased cardiac fibrosis in cold-treated mice, and Resveratrol treatment may inhibit cardiac fibrosis in cold-treated mice. Data were repeated three times. $\mathrm{n}=5$ mice in each group. Ctl, Control mice; Model, Cold-treated mice; Rsv, Cold-treated mice with Resveratrol administration. ${ }^{*} \mathrm{p}<0.05$ vs Control group, \# $\mathrm{p}<0.05$ vs Cold group,.

\section{Resveratrol improved cardiac function of cold-treated mice}

Damage of myocardial structure usually results in alteration of heart functions. We further investigated the effects of Resveratrol treatment on cardiac functions of cold-treated mice. As shown in Figure 4, echocardiography showed that cold-treated mice had a lower $\mathrm{EF}$ and FS value compared with control mice $(\mathrm{n}=6, \mathrm{p}<0.05)$, indicating the decline of cardiac functions. Mice with Resveratrol $(100 \mathrm{mg} / \mathrm{kg} /$ day) treatment showed the augment of EF value compared with cold-treated mice $(n=6, p<0.05)$. In addition, cold-treated mice also showed an increase of LVPWd, LVIDd and LVIDs compared with control mice, and Resveratrol treatment may suppress the increase of LVPWd in cold-treated mice ( $n=6, p<0.05$ ) (Fig. 4).

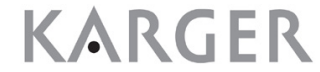




\section{Cellular Physiology and Biochemistry}

Fig. 3. Effects of Resveratrol on myocardial ultrastructure of cold-treated mice. TEM investigation showed that cellular ultrastructure was damaged in myocardium of cold-treated mice. Compared with control mice, the myofibrils were less organized in cold-treated mice. The loss of sarcomere registration, mitochondrial swollen and myofibrillar disarray were found in the myocardium of cold-treated mice. These morphological changes such as swollen mitochondria were reduced in cold-treated mice after Resveratrol treatment. The experiments were performed three times. $\mathrm{n}=3$ mice in each group. Ctl, Control mice; Model, Cold-treated mice; Rsv, Cold-treated mice with Resveratrol administration.

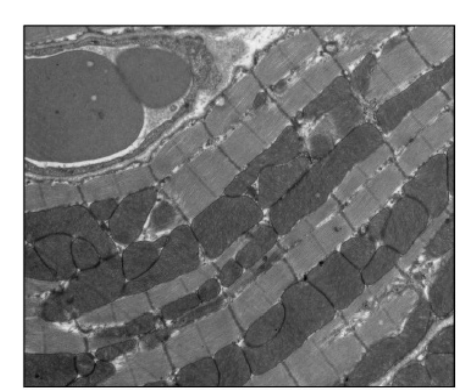

Ctl

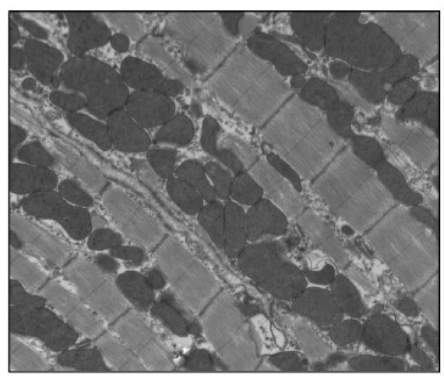

Rsv

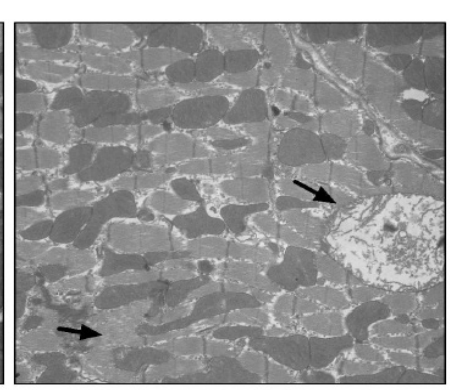

Model
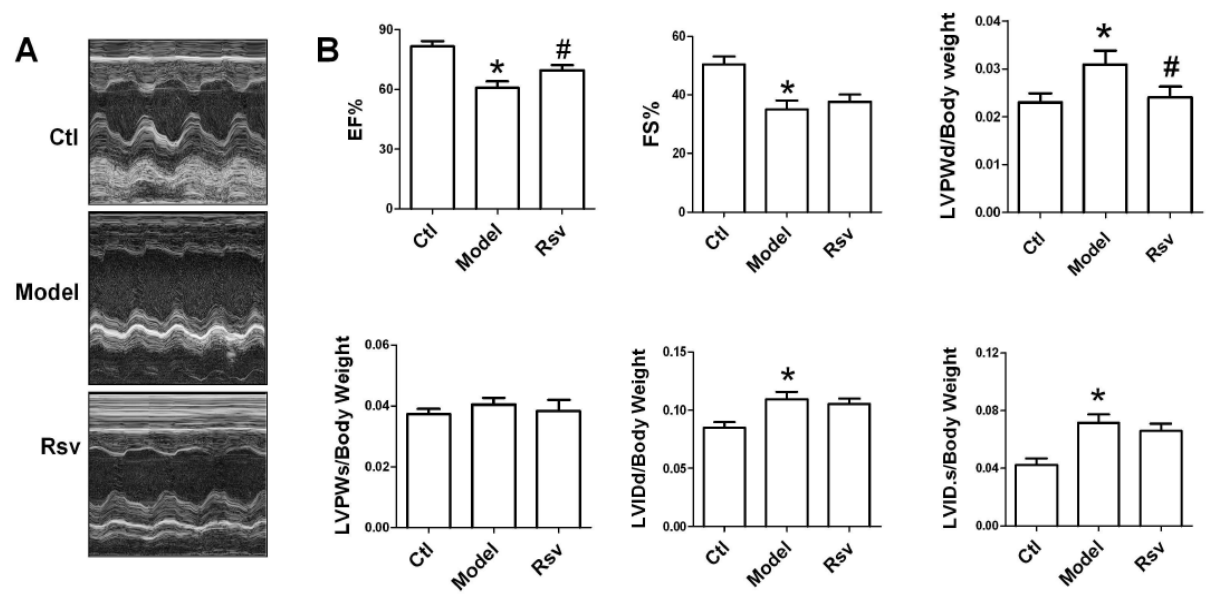

Fig. 4. Effects of Resveratrol on cardiac function of cold-treated mice. (A), The representative echocardiography pictures of left ventricle from control group, cold group and Resveratrol group. (B), Cold exposure induced a lower EF and FS value compared with control mice. Resveratrol treatment partially prevented the decline of EF in cold-treated mice. Cold exposure also induced the increase of LVPWd, LVIDd and LVIDs compared with control mice. Resveratrol treatment suppressed the increase of LVPWd in cold-treated mouse hearts. Data were repeated three times. $\mathrm{n}=6$ mice in each group. Ctl, Control mice; Model, Cold-treated mice; Rsv, Cold-treated mice with Resveratrol administration. ${ }^{*} \mathrm{p}<0.05$ vs Control group, \# $\mathrm{p}<0.05$ vs Cold group.

These results suggest that Resveratrol treatment produces benefits on cardiac functions in cold-treated mice.

Resveratrol suppressed cold-induced hypertension in mice

Increasing evidence revealed that exposure to cold was able to induce hypertension in mice $[14,15]$. Therefore, we investigated if Resveratrol treatment can inhibit the progress 

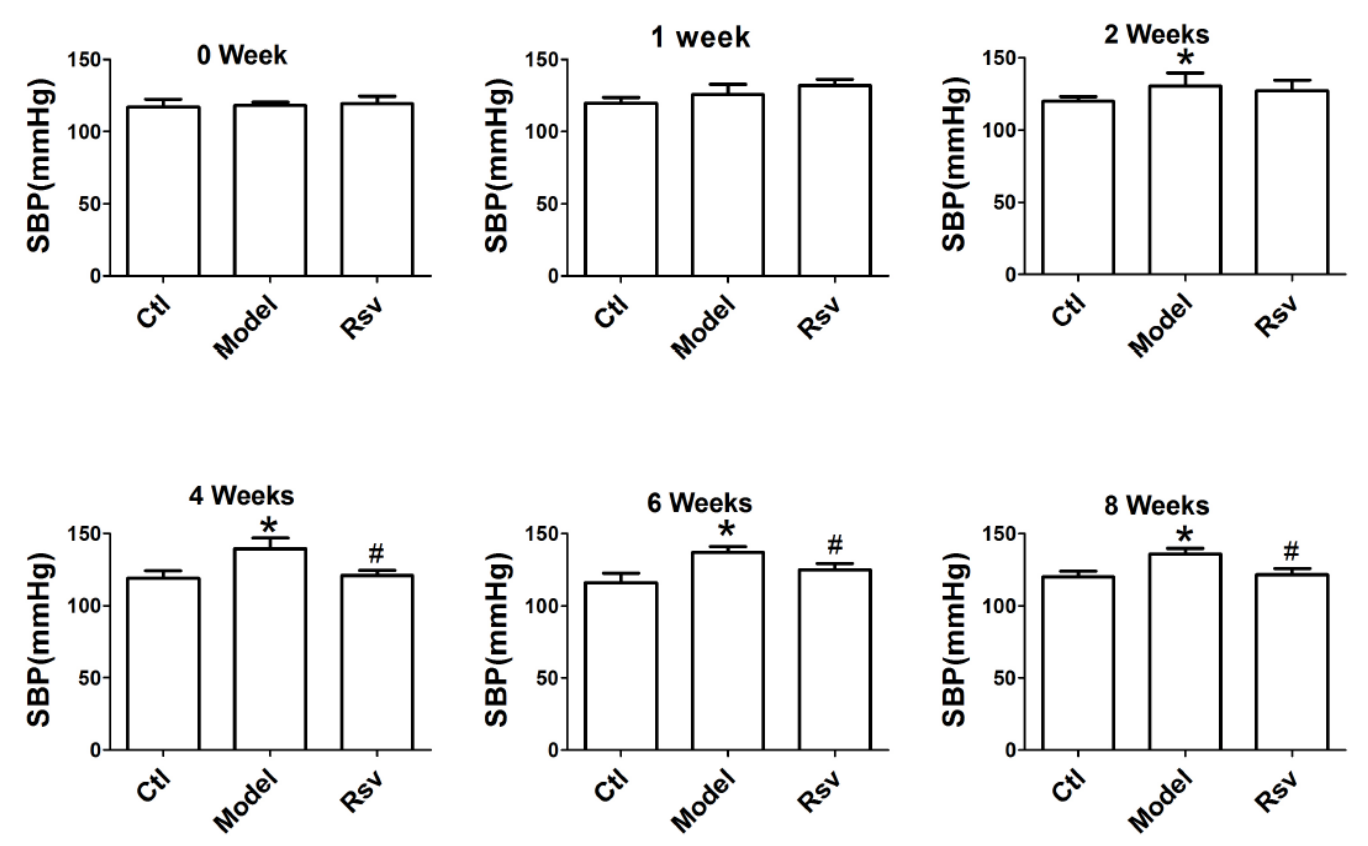

Fig. 5. Effects of Resveratrol on cold-induced hypertension in mice. Cold exposure led to the increase of systolic blood pressure (SBP) in mice after the second week. Resveratrol treatment can inhibit the augment of blood pressure in cold-treated mice. Ctl, Control mice; Model, Cold-treated mice; Rsv, Cold-treated mice with Resveratrol administration. The experiments were performed three times. $n=6$ mice in each group. * $\mathrm{p}<0.05$ vs Control group, \# $\mathrm{p}<0.05$ vs Cold group.

of hypertension in mice after exposure to cold for 1, 2, 4, 6 and 8 weeks. Consistently with previous studies $[14,15]$, mice exposed to cold displayed a higher blood pressure from the second week to the eighth week as compared to control mice $(n=6, p<0.05)$ (Fig. 5). However, treatment with Resveratrol $(100 \mathrm{mg} / \mathrm{kg} /$ day $)$ in mice showed a decrease of blood pressure compared with cold-treated mice $(n=6, p<0.05)$. It suggests that Resveratrol may attenuate cold-induced hypertension in mice.

\section{Resveratrol did not affect MAPK signal pathway}

It is well documented that MAPK signal pathway contributes to myocardial hypertrophy induced by certain stimuli such as isoproterenol infusion [29]. Whether p38, ERK and JNK signals are altered in cold-induced hypertrophic myocardium has not been elucidated yet. In this study, we also test if p38, ERK and JNK are involved in myocardium from cold-treated mice. We found that the expression of p-JNK, p-p38, p-ERK, T-JNK, T-p38 and T-ERK in cold group was not significantly changed as compared to control group $(n=4, p>0.05)$ (Fig. 6). Also, Resveratrol $(100 \mathrm{mg} / \mathrm{kg} /$ day) treatment did not obviously affect the expression of p-JNK, p-p38, p-ERK, T-JNK, T-p38 and T-ERK in mice $(n=4, p>0.05)$. It showed that MAPK signal did not contribute to the prevention of cold-induced heart hypertrophy by Resveratrol.

\section{Resveratrol inhibited the increase of miR-328 in cold-treated mouse hearts}

MicroRNAs (miRNAs) play an important role in the regulation of proliferation, apoptosis and aging of cardiomyocytes [30,31]. Previous study has showed that overexpression of miR-328 and miR-23a is able to induce myocardial hypertrophy $[28,31]$. We therefore measured the level of miR-328 and miR-23a among the three groups. Figure 7 showed that miR-328 level was significantly upregulated in cold-treated mouse hearts compared with control mice $(n=4, p<0.05)$. It indicates that miR-328 contributes to cold-induced myocardial remodeling. Furthermore, Resveratrol treatment can suppress the increase of miR-328 level 


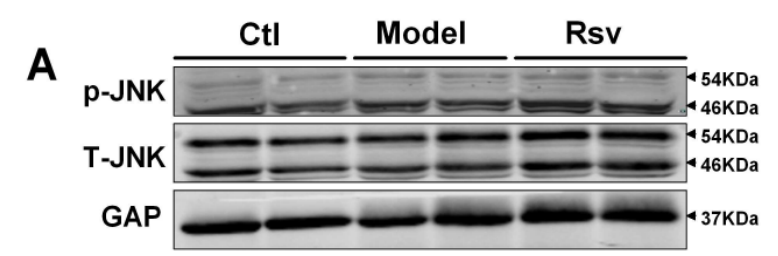

B

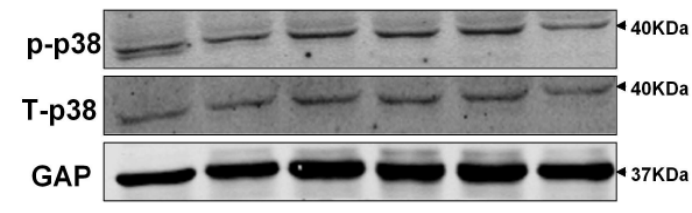

C

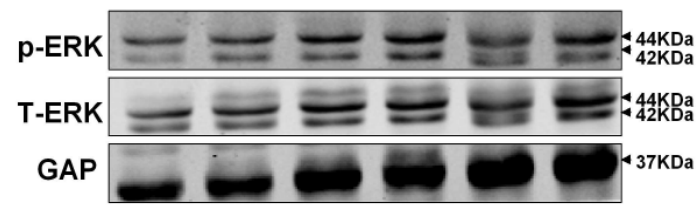

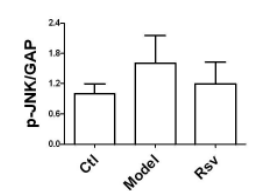
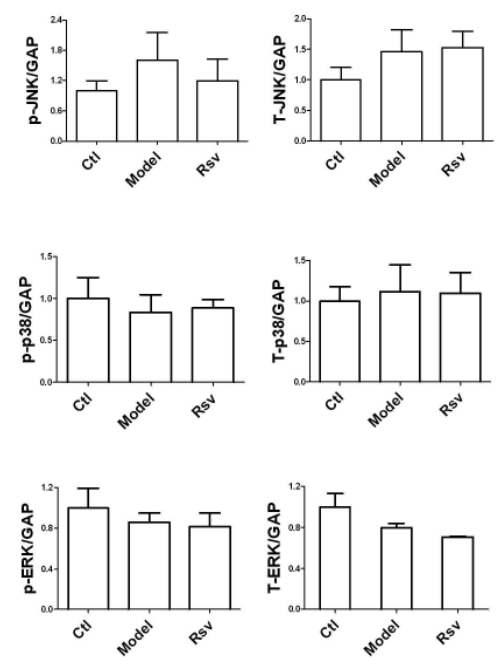

Fig. 6. Effects of Resveratrol on MAPK signal pathway in cold-treated mouse hearts. There was no significant difference of expression of p-JNK, p-p38, p-ERK, T-JNK, T-p38 and T-ERK among control group and cold group. Resveratrol treatment also did not affect the expression of p-JNK, p-p38, p-ERK, T-JNK, T-p38 and T-ERK in cold-treated mice. Ctl, Control mice; Model, Cold-treated mice; Rsv, Cold-treated mice with Resveratrol administration. Data were repeated three times. $\mathrm{n}=4$ mice in each group. ${ }^{*} \mathrm{p}<0.05$ vs Control group, \# $\mathrm{p}<0.05$ vs cold group.
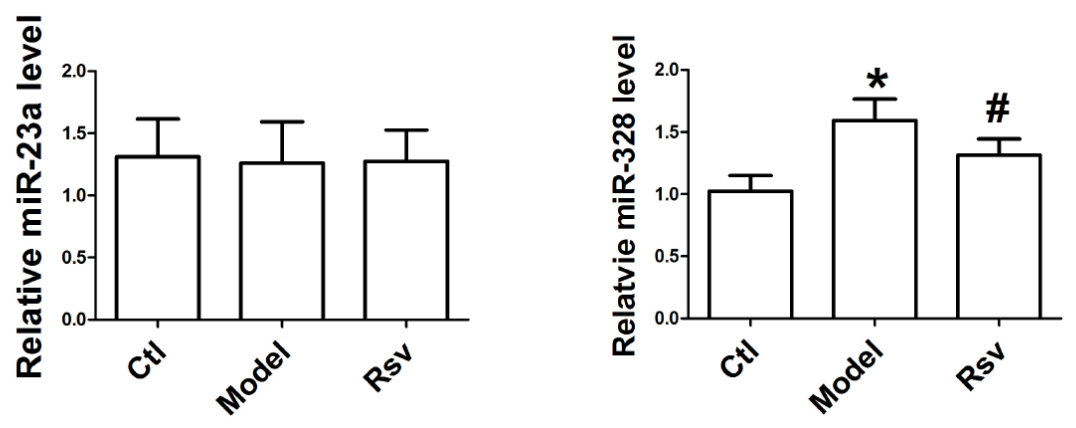

Fig. 7. Effects of Resveratrol on miR-328 and miR-23a in cold-treated mice. Cold-treated mouse hearts have an increase of miR-328 level compared with control mice, which can be effectively prevented by Resveratrol treatment. The miR-23a level was not altered in both cold group and Resveratrol group. Ctl, Control mice; Model, Cold-treated mice; Rsv, Cold-treated mice with Resveratrol administration. The experiments were performed three times. $\mathrm{n}=4$ mice in each group. ${ }^{*} \mathrm{p}<0.05$ vs Control group, $\# \mathrm{p}<0.05$ vs Cold group.

in cold-treated mice $(n=4, p<0.05)$. It suggests that miR-328 mediates the inhibition of coldinduced myocardium hypertrophy by Resveratrol treatment. Nevertheless, miR-23a level was not altered in cold-treated myocardium without or with Resveratrol treatment $(n=4$, p>0.05) (Fig. 7).

\section{Resveratrol suppressed apoptosis of myocardium of cold-treated mice}

The above data provided the evidence that exposure to cold is able to induce cardiovascular remodeling in mice including increase of blood pressure and cardiac structural remodeling. Previous studies have showed that apoptosis of cardiomyocytes was involved in cardiac remodeling in some heart diseases [32]. So we further investigate if cardiomyocyte apoptosis is also related to the changes of myocardial remodeling in cold-treated mice. As 


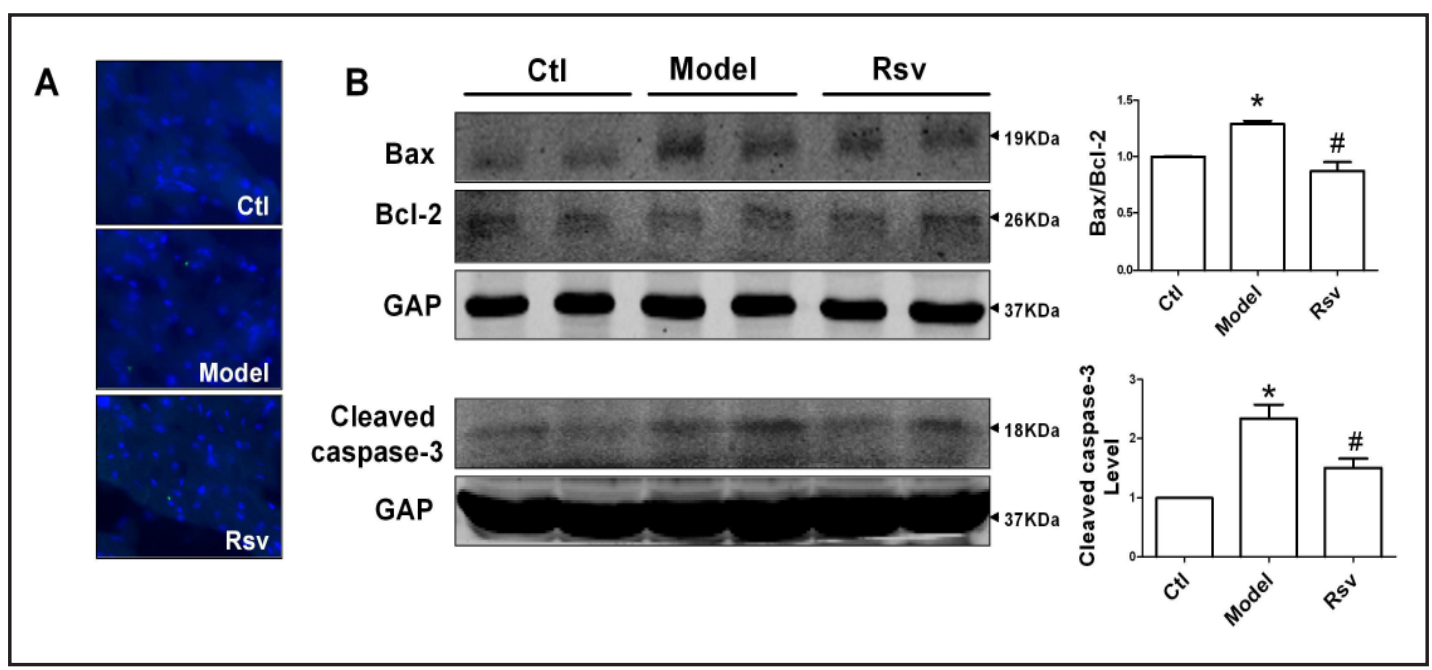

Fig. 8. Effects of Resveratrol on apoptosis of myocardium of cold-treated mice. (A), Compared with control group, cold-treated mouse heart showed an increase of apoptotic cells by TUNEL staining. But Resveratrol treatment may inhibit the increase of apoptotic cells in cold-treated mouse hearts. (B), Western blot confirmed that the ratio of Bax/Bcl-2 proteins was enhanced in cold-treated mouse hearts as compared with control mice, and Resveratrol treatment can suppress the increase of Bax/Bcl-2 ratio. The augment of cleaved caspase- 3 protein in cold-treated mouse hearts was inhibited by Resveratrol treatment as well. The experiments were performed three times. $n=4$ mice in each group. Ctl, Control mice; Model, Cold-treated mice; Rsv, Cold-treated mice with Resveratrol supplement. * p $<0.05$ vs Control group, \# p $<0.05$ vs Cold group.

shown in Figure 8A, TUNEL staining revealed that there was a slight increase of apoptotic cells in cold-treated mice compared with control mice. Resveratrol treatment may reduce the percentage of apoptotic cells in cold-treated mice. Furthermore, Western blot confirmed that the ratio of Bax/Bcl-2 proteins was augmented in cold-treated mice, and Resveratrol treatment may suppress this change in cold-treated mice $(n=4, p<0.05)$. Also, the augment of cleaved caspase- 3 protein in cold-treated mouse hearts was inhibited by Resveratrol treatment.

\section{Discussion}

For the first time, we uncovered that Resveratrol treatment may inhibit cold-induced heart structural and functional changes via suppressing miR-328 expression and apoptosis. This study provided more insights into molecular mechanisms of cold-related heart hypertrophy, and indicates dietary supplement of Resveratrol or Resveratrol-containing products as a potential strategy for the prevention and therapy of cold-related cardiovascular diseases.

Cardiovascular disease remains a major cause of people death all over the world, with the growing incidence year by year. Many factors such as genetics, diets and excise are shown to accord for the pathogenesis of cardiovascular diseases. Recent studies revealed that cold exposure was strongly linked with cardiovascular mortality $[1,5,6$, 9]. Conversely, a American epidemical study showed that an increase of temperature in fall, winter and spring was correlated with a reduction of cardiovascular deaths [2]. Cold stress was shown to induce cardiomyocyte apoptosis, hypertrophy and the decline of myocardial contractility $[8,12-14]$. So, low temperature stress was considered as a new potential risk factor of sudden cardiovascular death in human beings [7,9].

Consistently with previous studies [8], we also observed myocardial remodeling in mice with cold stress. Low ambient temperature is able to induce an increase of heart/ body weight index compared with control mice [12]. HE staining further demonstrated that 


\section{Cellular Physiology Cell Physiol Biochem 2015;35:2451-2462 \begin{tabular}{ll|l} 
aOI: 10.1159/000374045 & $\begin{array}{l}\text { O 2015 S. Karger AG, Basel } \\
\text { www.karger.com/cpb }\end{array}$ \\
\hline
\end{tabular} \\ Yin et al.: Resveratrol Inhibited Cold-Induced Heart Hypertrophy}

cold exposure resulted in the augment of cardiomyocyte size as characterized by increased cross-section surface area of cardiomyocytes. Cardiac fibrosis was also identified in mice with cold exposure using Masson staining. TEM was also used to detect the ultrastructure of myocardium from cold-treated mice, and found the abnormalities of mitochondria and myofibrils. These findings suggest that cold stress is able to damage myocardial structure and induce myocardial remodeling.

Alteration of myocardial structure often influences cardiac functions. As proposed, cardiac function was decreased in mice with cold exposure. This result is in coinciding with previous studies $[12,14]$. The MAPK signal pathway was showed to be involved in pathological heart hypertrophy induced by certain stimuli [29]. Nevertheless, we did not observe that JNK, p38 and ERK were markedly influenced in cold-treated mice in this study. But, we found that cold-treated mouse hearts have an increase of apoptotic cardiomyocytes compared with control mice. microRNAs has emerged as a novel important regulator of multiple physiological and pathological process including proliferation, apoptosis, differentiation and autophagy [28, 30,31]. Increasing evidence showed that miRNA imbalance was associated with pathological myocardial hypertrophy $[28,30]$. For instance, miR-23a and miR-328 have been shown to promote the development of myocardial hypertrophy, while miR-328 acted as an anti-hypertrophic factor in hearts [28, 30,31]. Overexpression of miR-328 resulted in cardiomyocyte hypertrophy via by inhibiting sarcoplasmic reticulum $\mathrm{Ca}^{2+}$ ATPase (SERCA2a) $[28,31]$. Upregulation of miR-23a is also able to induce hypertrophy in heart via targeting MuRF1 [28, 31]. In this study, we measured the level of miR-23a and miR-328 in mouse hearts with cold exposure, and the results displayed the increase of miR-328 but not miR-23a in cold-treated mice. These results suggest that the increase of miR-328 and cardiomyocyte apoptosis contributes to cold-induced myocardial remodeling.

Resveratrol as a heart-healthy compound is widely found in plants such as grape seed and skin, and also is an important natural substance in red wine [16, 23]. A large body of evidence has shown that Resveratrol has antiviral, antioxidant and anti-inflammatory actions, and may help prevent the development of certain cancers and cardiovascular diseases $[19,22]$. Resveratrol at a dosage of $146 \mathrm{mg} / \mathrm{kg} /$ day in rats may attenuate pathology left ventricular hypertrophy via inhibiting NFAT-dependent transcription [17]. Resveratrol treatment $(\sim 320 \mathrm{mg} / \mathrm{kg} /$ day $)$ also may suppress transverse aortic constriction (TAC)induced heart failure and reverse myocardial remodeling by restored cardiac AMP-activated protein kinase (AMPK) activation in mice [22]. Resveratrol treatment $(\sim 320 \mathrm{mg} / \mathrm{kg} /$ day in mice) may inhibit high blood pressure (BP) and prevent myocardial hypertrophy through mediating LKB1-AMPK-eNOS signaling axis [16]. Homocysteine-induced apoptosis of ventricular myocytes was also attenuated by Resveratrol treatment [33]. Oral supplement with $100 \mathrm{mg} / \mathrm{kg} /$ day of Resveratrol may improve ventricular function and increase blood flow to the myocardium remote from an area of ischemia [32]. In this study, we investigated the effects of Resveratrol $(100 \mathrm{mg} / \mathrm{kg} /$ day) treatment on myocardial hypertrophy induced by cold exposure. We found that Resveratrol treatment at $100 \mathrm{mg} / \mathrm{kg} /$ day is enough to inhibit the enlargement of cardiomyocyte size and cardiac apoptosis in cold-treated mice. This result is consistent with the benefits of Resveratrol on other heart diseases. The ultrastructure damage in cold-treated myocardium was also improved by Resveratrol treatment. Then, the decline of heart functions including EF and LVIDd in mice with cold stress was partially restored by Resveratrol treatment. Lots of studies have reported that cardiomyocyte apoptosis was associated with myocardial remodeling in heart diseases [32]. We found that Resveratrol treatment also inhibited the apoptosis of cardiomyocytes of cold-treated mice. But Resveratrol treatment did not impact the activation of JNK, p38 and ERK signal in coldtreated mouse hearts. Furthermore, we found that Resveratrol treatment partially revered the increase of miR-328 in myocardium from mice with cold stress. It suggested miR-328 mediates anti-hypertrophic action of Resveratrol on cold-induced myocardial hypertrophy.

In this study, we also found that cold-treated mice showed a significant incrase of systolic blood pressure, and Resveratrol treatment can prevent cold-induced blood pressure. Previous studies have uncovered that the activation of eNOS and NO production as well as 
the inhibitor of ET-1 and ETA receptor has been suggested as the therapeutic agents for coldinduced hypertension [10]. A recent study showed that Resveratrol can reduce oxidative stress, improve vascular function and attenuate high blood pressure through increasing endothelial NO synthase (eNOS) phosphorylation [16]. So we speculated that Resveratrol protected against cold-induced hypertension via activating eNOS signal pathway.

In summary, low ambient temperature may cause myocardial structural and functional remodeling and Resveratrol, a natural compound in plants such as grapes can effectively inhibit cold-induced myocardial hypertrophy. This finding suggests that dietary supplement of Resveratrol will protect hearts against cardiovascular diseases for people living in cold region.

\section{Conclusions}

Resveratrol suppressed low temperature-induced the enlarged heart size of mice, the damage of cardiac ultrastructure and the weakened function. The above changes may be associated with the inhibition of miR-328 expression and apoptosis.

\section{Acknowledgements}

This work was supported by the National Natural Science Fund of China (30900601/81170096), the Program for New Century Excellent Talents In Heilongjiang Provincial University (1252-NCET-013)

\section{Disclosure Statement}

The authors have declared that no competing interest exists.

\section{References}

1 Atsumi A, Ueda K, Irie F, Sairenchi T, Iimura K, Watanabe H, Iso H, Ota H, Aonuma K: Relationship between cold temperature and cardiovascular mortality, with assessment of effect modification by individual characteristics: Ibaraki prefectural health study. Circ J 2013;77:1854-1861.

2 Barnett AG: Temperature and cardiovascular deaths in the us elderly: Changes over time. Epidemiology 2007;18:369-372.

3 Tian Z, Li S, Zhang J, Jaakkola JJ, Guo Y: Ambient temperature and coronary heart disease mortality in beijing, china: A time series study. Environ Health 2012;11:56.

4 Donaldson GC, Keatinge WR: Early increases in ischaemic heart disease mortality dissociated from and later changes associated with respiratory mortality after cold weather in south east england. J Epidemiol Community Health 1997;51:643-648.

5 Raguz M, Bergovec M, Kranjcec D, Vrazic H, Puksic S: A cold shock response triggering acute myocardial infarction. Int J Cardiol 2008;128:e37-39.

6 Li Y, Alshaer H, Fernie G: Blood pressure and thermal responses to repeated whole body cold exposure: Effect of winter clothing. Eur J Appl Physiol 2009;107:673-685.

7 Cheng X, Su H: Effects of climatic temperature stress on cardiovascular diseases. Eur J Intern Med 2010;21:164-167.

8 Lu S, Xu D: Cold stress accentuates pressure overload-induced cardiac hypertrophy and contractile dysfunction: Role of trpv1/ampk-mediated autophagy. Biochem Biophys Res Commun 2013;442:8-15.

9 Cagle A, Hubbard R: Cold-related cardiac mortality in king county, washington, USA 1980-2001. Ann Hum Biol 2005;32:525-537.

10 Sun Z: Cardiovascular responses to cold exposure. Front Biosci (Elite Ed) 2010;2:495-503.

11 Chen GF, Sun Z: Effects of chronic cold exposure on the endothelin system. J Appl Physiol (1985) 2006;100:1719-1726.

12 Bello Roufai M, Li H, Sun Z: Heart-specific inhibition of protooncogene c-myc attenuates cold-induced cardiac hypertrophy. Gene Ther 2007;14:1406-1416. 


\section{Cellular Physiology Cell Physiol Biochem 2015;35:2451-2462 \begin{tabular}{l|l} 
DOI: 10.1159/000374045 & (C) 2015 S. Karger AG, Basel
\end{tabular} and Biochemistry Published online: April 24, $2015 \quad$ www.karger.com/cpb \\ Yin et al.: Resveratrol Inhibited Cold-Induced Heart Hypertrophy}

13 Zhang Y, Li L, Hua Y, Nunn JM, Dong F, Yanagisawa M, Ren J: Cardiac-specific knockout of et(a) receptor mitigates low ambient temperature-induced cardiac hypertrophy and contractile dysfunction. J Mol Cell Biol 2012;4:97-107.

14 Sun Z, Cade R, Zhang Z, Alouidor J, Van H: Angiotensinogen gene knockout delays and attenuates coldinduced hypertension. Hypertension 2003;41:322-327.

15 Wang X, Skelley L, Cade R, Sun Z: Aav delivery of mineralocorticoid receptor shrna prevents progression of cold-induced hypertension and attenuates renal damage. Gene Ther 2006;13:1097-1103.

16 Dolinsky VW, Chakrabarti S, Pereira TJ, Oka T, Levasseur J, Beker D, Zordoky BN, Morton JS, Nagendran J, Lopaschuk GD, Davidge ST, Dyck JR: Resveratrol prevents hypertension and cardiac hypertrophy in hypertensive rats and mice. Biochim Biophys Acta 2013;1832:1723-1733.

17 Dolinsky VW, Soltys CL, Rogan KJ, Chan AY, Nagendran J, Wang S, Dyck JR: Resveratrol prevents pathological but not physiological cardiac hypertrophy. J Mol Med (Berl) 2014

18 Ma W, Zhao L, Yin K, Feng D, Yang F, Liang J, Chen H, Bi C, Li X, Wang Y, Cai B: Effects of arsenic trioxide on proliferation, paracrine and migration of cardiac progenitor cells. Int J Cardiol 2015;179:393-396.

19 Garcia-Zepeda SP, Garcia-Villa E, Diaz-Chavez J, Hernandez-Pando R, Gariglio P: Resveratrol induces cell death in cervical cancer cells through apoptosis and autophagy. Eur J Cancer Prev 2013;22:577-584.

20 Fouad MA, Agha AM, Merzabani MM, Shouman SA: Resveratrol inhibits proliferation, angiogenesis and induces apoptosis in colon cancer cells: Calorie restriction is the force to the cytotoxicity. Hum Exp Toxicol 2013;32:1067-1080.

21 Luo H, Yang A, Schulte BA, Wargovich MJ, Wang GY: Resveratrol induces premature senescence in lung cancer cells via ros-mediated DNA damage. PLoS One 2013;8:e60065.

22 Sung MM, Das SK, Levasseur J, Byrne NJ, Fung D, Kim T, Masson G, Boisvenue J, Soltys CL, Oudit GY, Dyck JR: Resveratrol treatment of mice with pressure overload-induced heart failure improves diastolic function and cardiac energy metabolism. Circ Heart Fail 2015;8:128-137.

23 Chen YR, Yi FF, Li XY, Wang CY, Chen L, Yang XC, Su PX, Cai J: Resveratrol attenuates ventricular arrhythmias and improves the long-term survival in rats with myocardial infarction. Cardiovasc Drugs Ther 2008;22:479-485.

24 Cai B, Wang G, Chen N, Liu Y, Yin K, Ning C, Li X, Yang F, Wang N, Wang Y, Pan Z, Lu Y: Bone marrow mesenchymal stem cells protected post-infarcted myocardium against arrhythmias via reversing potassium channels remodelling. J Cell Mol Med 2014;18:1407-1416.

25 Yasumoto K, Takata M, Ueno H, Tomita S, Tomoda F, Inoue H: Relation of plasma brain and atrial natriuretic peptides to left ventricular geometric patterns in essential hypertension. Am J Hypertens 1999;12:921924.

26 Biala A, Finckenberg P, Korpi A, Loytainen M, Martonen E, Levijoki J, Mervaala E: Cardiovascular effects of the combination of levosimendan and valsartan in hypertensive dahl/rapp rats. J Physiol Pharmacol 2011;62:275-285.

27 Vaingankar SM, Li Y, Corti A, Biswas N, Gayen J, O'Connor DT, Mahata SK: Long human chga flanking chromosome 14 sequence required for optimal bac transgenic "Rescue" Of disease phenotypes in the mouse chga knockout. Physiol Genomics 2010;41:91-101.

28 Li C, Li X, Gao X, Zhang R, Zhang Y, Liang H, Xu C, Du W, Liu X, Ma N, Xu Z, Wang L, Chen X, Lu Y, Ju J, Yang B, Shan H: Microrna-328 as a regulator of cardiac hypertrophy. Int J Cardiol 2014;173:268-276.

29 Muslin AJ: Mapk signalling in cardiovascular health and disease: Molecular mechanisms and therapeutic targets. Clin Sci (Lond) 2008;115:203-218.

30 Ganesan J, Ramanujam D, Sassi Y, Ahles A, Jentzsch C, Werfel S, Leierseder S, Loyer X, Giacca M, Zentilin L, Thum T, Laggerbauer B, Engelhardt S: Mir-378 controls cardiac hypertrophy by combined repression of mitogen-activated protein kinase pathway factors. Circulation 2013;127:2097-2106.

31 Lin Z, Murtaza I, Wang K, Jiao J, Gao J, Li PF: Mir-23a functions downstream of nfatc3 to regulate cardiac hypertrophy. Proc Natl Acad Sci U S A 2009;106:12103-12108.

32 Qin F, Siwik DA, Pimentel DR, Morgan RJ, Biolo A, Tu VH, Kang YJ, Cohen RA, Colucci WS: Cytosolic $\mathrm{H}_{2} \mathrm{O}_{2}$ mediates hypertrophy, apoptosis, and decreased serca activity in mice with chronic hemodynamic overload. Am J Physiol Heart Circ Physiol 2014;306:H1453-1463.

33 Liu Y, Liu Q, Hu Z, Shen Q, Liang H, Jiang X: Resveratrol attenuated homocysteine-induced apoptosis of rat ventricular myocytes. Int J Cardiol 2014;177:298-300. 